\title{
Single-Molecule Vacuum Rabi Splitting: Four-Wave Mixing and Optical Switching at the Single-Photon Level
}

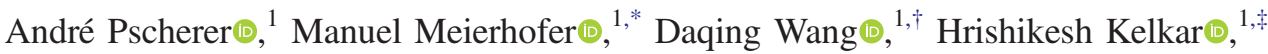 \\ Diego Martín-Cano®, ${ }^{1,8}$ Tobias Utikal $\odot,{ }^{1}$ Stephan Götzinger $\odot,{ }^{2,1,3}$ and Vahid Sandoghdar $\circledast^{1,2}$ \\ ${ }^{1}$ Max Planck Institute for the Science of Light, D-91058 Erlangen, Germany \\ ${ }^{2}$ Department of Physics, Friedrich-Alexander University Erlangen-Nürnberg (FAU), \\ D-91058 Erlangen, Germany \\ ${ }^{3}$ Graduate School in Advanced Optical Technologies (SAOT), Friedrich-Alexander University Erlangen-Nürnberg, \\ Erlangen D-91052, Germany
}

(Received 4 May 2021; accepted 12 August 2021; published 24 September 2021)

\begin{abstract}
A single quantum emitter can possess a very strong intrinsic nonlinearity, but its overall promise for nonlinear effects is hampered by the challenge of efficient coupling to incident photons. Common nonlinear optical materials, on the other hand, are easy to couple to but are bulky, imposing a severe limitation on the miniaturization of photonic systems. In this Letter, we show that a single organic molecule acts as an extremely efficient nonlinear optical element in the strong coupling regime of cavity quantum electrodynamics. We report on single-photon sensitivity in nonlinear signal generation and all-optical switching. Our work promotes the use of molecules for applications such as integrated photonic circuits operating at very low powers.
\end{abstract}

DOI: 10.1103/PhysRevLett.127.133603

The cross section of materials for nonlinear optical processes is known to be small so that measurements are usually performed under intense laser illumination [1,2]. Considering that the linear cross section of a single twolevel atom $\left(\sigma_{0}=3 \lambda^{2} / 2 \pi, \lambda\right.$ is the transition wavelength) is large enough to result in the complete extinction of an optical beam [3], one might wonder about the ability of an atom or a molecule to generate nonlinear signals with single-photon sensitivity [4]. It turns out, however, that $\sigma_{0}$ for real-life quantum emitters is compromised by the influence of many transition paths, dissipation, or dephasing [5]. To overcome the resulting decrease in coupling efficiency, single emitters such as cold alkali atoms [6], semiconductor quantum dots [7-9], or color centers [10,11] have been investigated in the strong-coupling regime of cavity quantum electrodynamics (CQED).

Although organic molecules were among the first nonlinear optical media that were exploited $[12,13]$, they have been under-represented in nonlinear CQED studies: strong coupling has been reported for ensembles of molecules $[14,15]$ and, in one claim, with single-molecule sensitivity but at a low degree of coherence [16]. In this Letter, we

Published by the American Physical Society under the terms of the Creative Commons Attribution 4.0 International license. Further distribution of this work must maintain attribution to the author(s) and the published article's title, journal citation, and DOI. Open access publication funded by the Max Planck Society. present the first case of strong coupling between a Fourierlimited single molecule and a microcavity. We demonstrate the high efficiency of this medium for coherent nonlinear optical interactions in three concrete studies of saturation, four-wave mixing and its higher harmonics, and optical switching, all down to the single photon level.

The molecule in our current work is dibenzoterrylene (DBT) from the family of polycyclic aromatic hydrocarbons. As in the case of other organic dye molecules, the excited state in DBT can decay via a manifold of vibrational levels $|g, v=0,1,2, .$.$\rangle in the electronic ground$ state [see inset in Fig. 1]. When embedded in an appropriate organic crystal such as anthracene (AC), the zero-phonon line (00ZPL) associated with the transition between $\mid g, v=$ $0\rangle$ and $|e, v=0\rangle$ boasts a Fourier-limited linewidth $(\gamma)$ at liquid helium temperature $[17,18]$. The total dipole moment associated with the decay of the excited state is estimated to be $13 \mathrm{D}$ [19]. The branching ratio, defined as the ratio of the power emitted via the 00ZPL to the total fluorescence power, amounts to about $30 \%$. To compensate for the loss of coherence through the red-shifted decay channels, we recently coupled a DBT:AC sample to a scanning FabryPerot microcavity and demonstrated that the composite molecule-cavity system behaves like a coherent two-level system [17]. In this Letter, we extend that work by entering the strong coupling regime of CQED and explore nonlinear interactions such as four-wave mixing and optical switching at the quantum level.

The experimental setup, including a cryostat and various optical and electronical components, was mostly as 


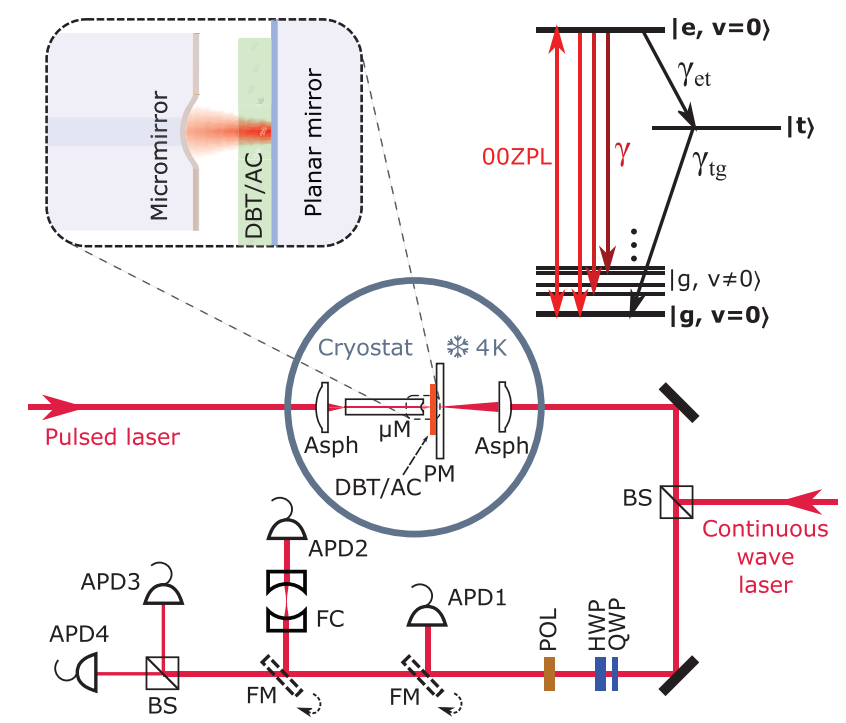

FIG. 1. Schematics of the experimental setup. Details can be found in Refs. [17,20] and the SM [21]. An external Fabry-Perot cavity (FC) serves as a narrow-band filter for the molecular emission. BS: beam splitter; Asph: aspheric lens; $\mu \mathrm{M}$ : micromirror; PM: planar mirror; DBT/AC: dibenzoterrylene-doped anthracene crystal; QWP: quarter-wave plate; HWP: half-wave plate; POL: polarization filter; FM: flip mirror; APD: avalanche photodiode. Left inset: An enlargement of the microcavity. Right inset: Jablonski diagram for DBT. The 00ZPL takes place at a wavelength of $\lambda \sim 785 \mathrm{~nm}$. $\gamma$ stands for the total decay rate of the excited state. The triplet state is denoted by $|t\rangle$.

described in Refs. [17,20] and is elaborated on in the Supplemental Material (SM) [21]. Here, it suffices to state that we use a wavelength-sized Fabry-Perot resonator consisting of a planar mirror and a second curved mirror, which is nanofabricated at the end of an optical fiber. The mirrors surround a thin DBT:AC crystal in the cryostat (see inset in Fig. 1). To probe the transmission response of the cavity, we examined the cross-polarized signal of the light reflected from the flat mirror side or directly detected the transmission of light coupled from the fiber side $[17,20]$. We accessed the strong coupling regime by exploiting the knowledge that the finesse of our current cavity is limited by residual mechanical instabilities (in the frequency range of $10 \mathrm{~Hz}-10 \mathrm{kHz}$ ), which remain after the cavity frequency is locked using a separate laser beam [17,20]. We thus synchronized our photon detection events with the locking error signal of the cavity to only record data in time intervals, where the cavity frequency $\left(\nu_{c}\right)$ coincides with the molecular 00ZPL frequency $\left(\nu_{m}\right)$ (see Section. IIIA in the SM [21]).

Figure 2(a) displays a transmission spectrum of the microcavity as the light from a narrow-band continuouswave titanium sapphire (Ti:Sapph) laser was coupled to the microcavity from its flat mirror end and the laser frequency was scanned. We find a Lorentzian cavity resonance with full width at half-maximum (FWHM) of $\kappa / 2 \pi=1.3 \mathrm{GHz}$ when detuned from a molecular line. To characterize the
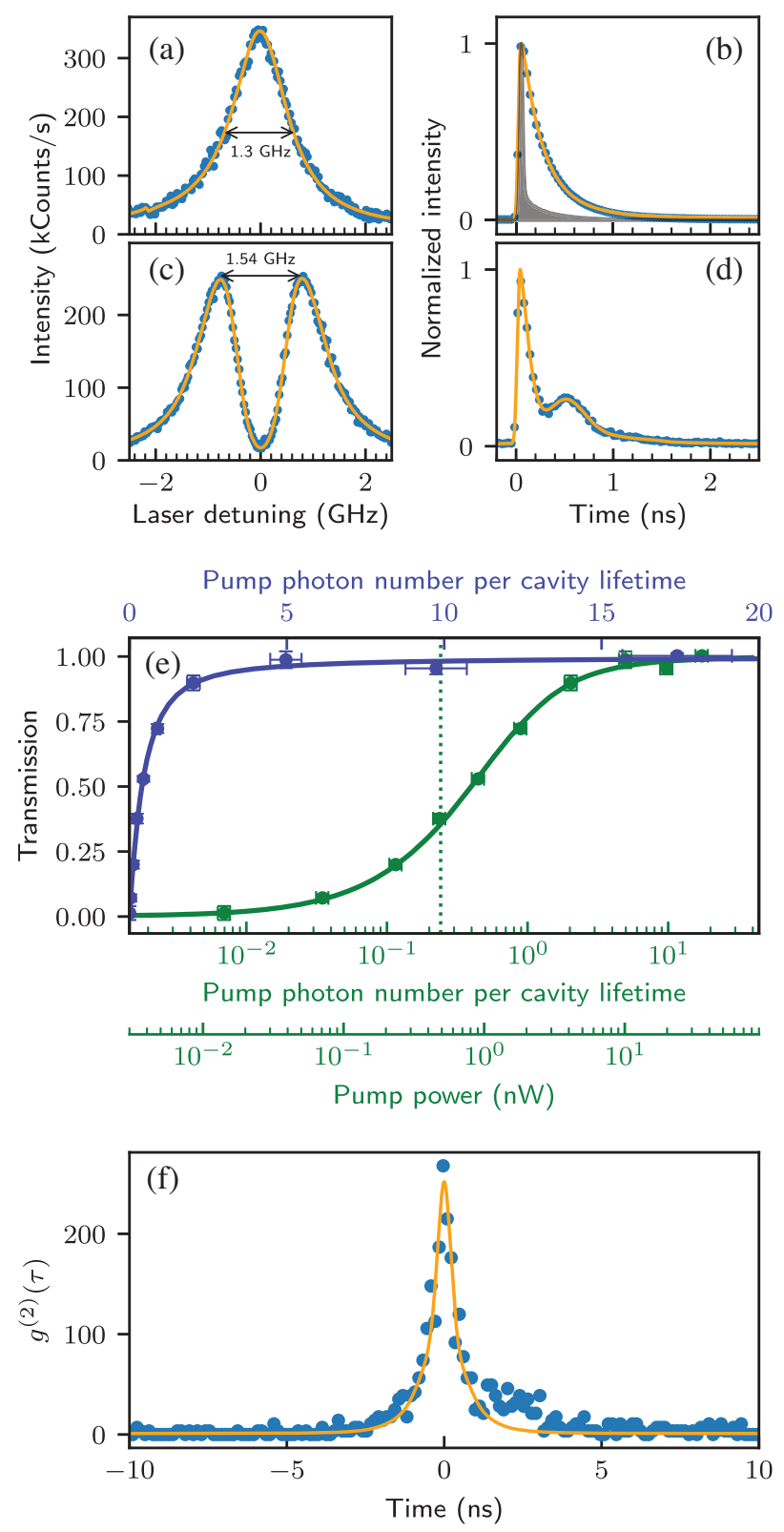

FIG. 2. Transmission spectrum of the bare cavity (a) and the coupled molecule-cavity system (c). Ring-down temporal signal of the bare cavity (b) and the coupled molecule-cavity system (d). See the SM [21] for information on the influence of the detector response time [shown by the gray area in (b)] and on the fits to the experimental data (solid orange curves). (e) Transmission through the cavity on resonance with the molecule and the laser as a function of the excitation power. Green and blue present the same data for different horizontal axis scalings. The vertical dotted line marks $S=1$. (f) Intensity autocorrelation of the light transmitted through the cavity on resonance with the molecule and the laser. The maximum value of 250 is limited by residual background light, which was accounted for by the theoretical fit (orange curve). The side peak expected at a delay of about 0.6 ns caused by the Rabi oscillation is washed out since the cavity frequency was not stabilized in this measurement to avoid residual background from the lock laser and due to the contribution of the detector response function (see Fig. S2 in the SM [21]). 
cavity further, we also performed ring-down measurements by exciting the cavity from its optical fiber side with a picosecond pulsed Ti:Sapph laser. Figure 2(b) shows the exponential decay of the intracavity power, which yields an $e^{-1}$ decay time of $125 \mathrm{ps}$ after deconvolving the instrument response function of the detector. The result matches very well the value $1 /(2 \pi \times 1.3 \mathrm{GHz})$ deduced from the linewidth of its Fourier transform. We point out in passing that this decay time is considerably shorter than the excitedstate lifetime of about $4 \mathrm{~ns}$ for DBT, i.e., $\kappa \gg \gamma \approx 2 \pi \times$ $40 \mathrm{MHz}$ [17].

If we now tune the cavity resonance to the 00ZPL of a single DBT molecule, the transmission spectrum features a vacuum Rabi splitting with $2 g / 2 \pi=1.54 \mathrm{GHz}$, as displayed in Fig. 2(c). In this measurement, we accounted for a Gaussian distribution of $0.90 \mathrm{GHz}$ in $\nu_{c}$ caused by residual vibrations that could not be eliminated in postprocessing (see Section. IIIA in the SM [21]). The corresponding timedomain measurement is shown in Fig. 2(d), where an oscillation is superimposed on the exponential cavity ringdown curve. The analysis of this signal yields a period of $656 \mathrm{ps}$, corresponding to a frequency of $1.53 \mathrm{GHz}$ and $\kappa=1.3 \mathrm{GHz}$. The time-resolved oscillations provide clear evidence that the observed splitting is indeed due to a coherent exchange of energy between the molecule and the cavity field, which is the hallmark of strong coupling in CQED [22]. The measurements presented in Fig. 2 let us extract the cooperativity parameter $C=4 g^{2} / \kappa \gamma=45$. Technical improvements in our microcavity setup will yield larger cooperativities in the near future. We note that anticrossing in transmission spectra does not provide robust evidence for reaching the strong coupling regime since it is also present in the weak coupling case [17]. Indeed, anticrossing spectra have usually been reported in the fluorescence signal, which is blocked by the mirrors in our arrangement. Furthermore, because $g<\kappa$, we do not resolve the Jaynes-Cummings ladder [23] in our system (see also Section. IIIB in the SM [21]).

Having established the regime of strong coupling, we now discuss the nonlinearity of the system. The simplest signature of nonlinearity in light-matter interactions stems from saturation, which is related to the intrinsic anharmonicity of a two-level system [2]. The green symbols in Fig. 2(e) display the cavity-molecule transmission on resonance as a function of the incident power. The solid green curve plots the results of numerical simulations based on parameters extracted from the measurements shown in Figs. 2(c),(d) and a careful calibration of the incident power (see the SM [21]). We reach a very good agreement with the experimental results with only the ratio of the intersystem crossing rates to $\left(\gamma_{\mathrm{et}}\right)$ and out of $\left(\gamma_{\mathrm{tg}}\right)$ the triplet state (see inset in Fig. 1) as a fit parameter [18,24]. As seen from the upper green horizontal axis in Fig. 2(e), the moleculecavity system experiences a saturation parameter of $S=1$ for an incident average photon number as low as
0.24 per cavity lifetime. Here, we have defined $S=$ $\left[\rho_{e e}\left(I_{\text {in }} \rightarrow \infty\right) / \rho_{e e}\left(I_{\text {in }}\right)-1\right]^{-1}$ where $\rho_{e e}$ denotes the excited state population at excitation intensity $I_{\text {in }}$ (see Section. IIIB in the SM [21]). We note that the observed behavior is similar to that of a common saturation curve from a bare molecule [25] when plotted on a linear scale [see the blue dataset in Fig. 2(e)].

Another interesting consequence of the strong nonlinear response of the molecule-cavity composite is expressed by the photon statistics of the transmitted light. Figure 2(f) displays the second-order autocorrelation function $g^{(2)}(\tau)$ measured in the weak excitation regime. The observed impressive superbunching stems from the difference in the response of the molecule-cavity system to different Fock state components $|N\rangle=|1\rangle,|2\rangle,|3\rangle$, etc., of the laser beam $[17,26,27]$. The strong response of the molecule to a singlephoton state can be exploited for photon sorting [28,29].

The results discussed in Fig. 2 establish molecular CQED on the same footing as alkali atoms, semiconductor quantum dots, and color center systems. We now present two investigations where a single molecule mediates the interaction between two laser beams with average photon numbers $\bar{N} \sim 1$. First, we explore four-wave mixing (FWM) as a common nonlinear optical phenomenon. To do this, we used an acousto-optical modulator to produce two laser beams at frequencies $\nu_{1}$ and $\nu_{2}$, which were symmetrically detuned around $\nu_{c}$ by $\Delta \nu / 2=150 \mathrm{MHz}$. The powers of the two beams were equalized and their frequencies set symmetrically on each side of $\nu_{c}$ (see inset in Fig. 3). We then cocoupled the laser beams to the microresonator and scanned an external filter cavity with a linewidth of $30 \mathrm{MHz}$ (see FC in Fig. 1) to probe the spectrum of the light exiting the molecule-cavity system. The orange data points in Fig. 3 show that already at a very low cavity-coupled power of $425 \mathrm{pW}$ per beam, corresponding to 0.21 photons per cavity lifetime, we observe the conversion of a pair of photons at frequencies $\left(\nu_{1}, \nu_{2}\right)$ to a pair at frequencies $\left(\nu_{1}-\Delta \nu, \nu_{2}+\Delta \nu\right)$ with an efficiency (ratio of FWM peak to main peak) of $1.4 \pm 0.3 \%$. This is substantially more efficient than our previous evidence of FWM produced by a molecule in a tight focus [4]. The measurements in Fig. 3 confirm that increasing the incident intensity lowers the efficiency since the interaction becomes less coherent for large saturation parameters $[2,25,30]$. Nevertheless, the absolute power in the FWM frequencies increases beyond $S=1$. In fact, we show that for excitation beyond saturation (1.7 $\mathrm{nW}$ per beam), a single molecule generates a six-photon process, corresponding to the detection of the second-order harmonics (see Fig. 3). In future efforts, it would be interesting to scrutinize the photons generated in the harmonics more closely to reveal their spatiotemporal entanglement [31]. Furthermore, the efficiency of the FWM process could be enhanced by synchronizing the incident photons [32], e.g., through the use of triggered photon guns [33]. 


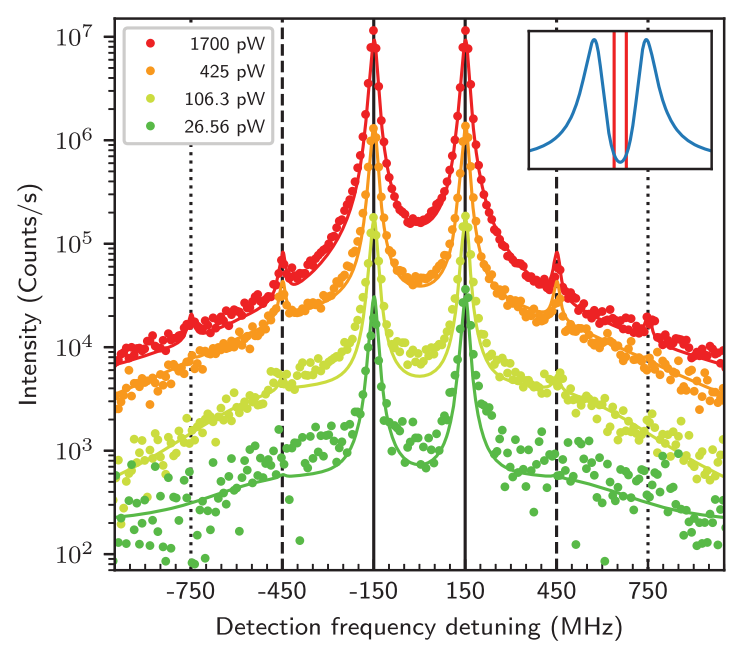

FIG. 3. Transmission spectrum of the coupled system under excitation at two frequencies separated by $300 \mathrm{MHz}$ at different powers (see legend). The spectra reveal the generation of higher harmonics. The theory curves reproduce the features. The vertical lines point to the regular frequency spacing of the observed signals.

Another powerful technique in nonlinear optics is to control the interaction between a medium and one light field (probe) via a second optical beam (pump). This approach is commonly employed in spectroscopy [1,32], but it is also encountered in signal processing schemes, where a gate beam is used to manipulate a signal beam [34,35]. To explore the latter scenario, we tuned the frequency of the weaker probe beam to $\nu_{c}=\nu_{m}$, while the pump frequency was detuned by $300 \mathrm{MHz}$ [see inset in Fig. 4(a)]. The transmission of the probe beam was then measured by scanning the filter cavity. The symbols in Fig. 4(a) display this quantity as a function of the pump power. The solid curve presents a very good agreement between the theoretical predictions and the experimental data based on $\gamma / 2 \pi=0.04 \mathrm{GHz}$ and independently measured parameters, $g / 2 \pi=0.63 \mathrm{GHz}, \kappa / 2 \pi=1.3 \mathrm{GHz}$, and the incoupling efficiency of $18 \%$. As in the case of Fig. 2(e), we left the ratio of the intersystem crossing rates $\left(\gamma_{\mathrm{et}} / \gamma_{\mathrm{tg}}\right)$ as a free fit parameter (see the SM [21]). Figure 4(a) shows that with only one photon per cavity lifetime, we can nearly fully turn on the probe beam transmission, which is otherwise blocked by the molecule. We remark that the increase of probe transmission beyond the value of $100 \%$ stems from the transfer of energy between the two beams [4].

The switching contrast, defined as the ratio of the transmitted powers with and without the pump, amounts to $40 \pm 17$ in Fig. 4(a), which is about 30 times higher than the best previous reports without a cavity $[4,35]$. The uncertainty in this quantity is dominated by the fluctuations in the low transmitted signal at weak pump powers. To compare our study to the system response for other choices
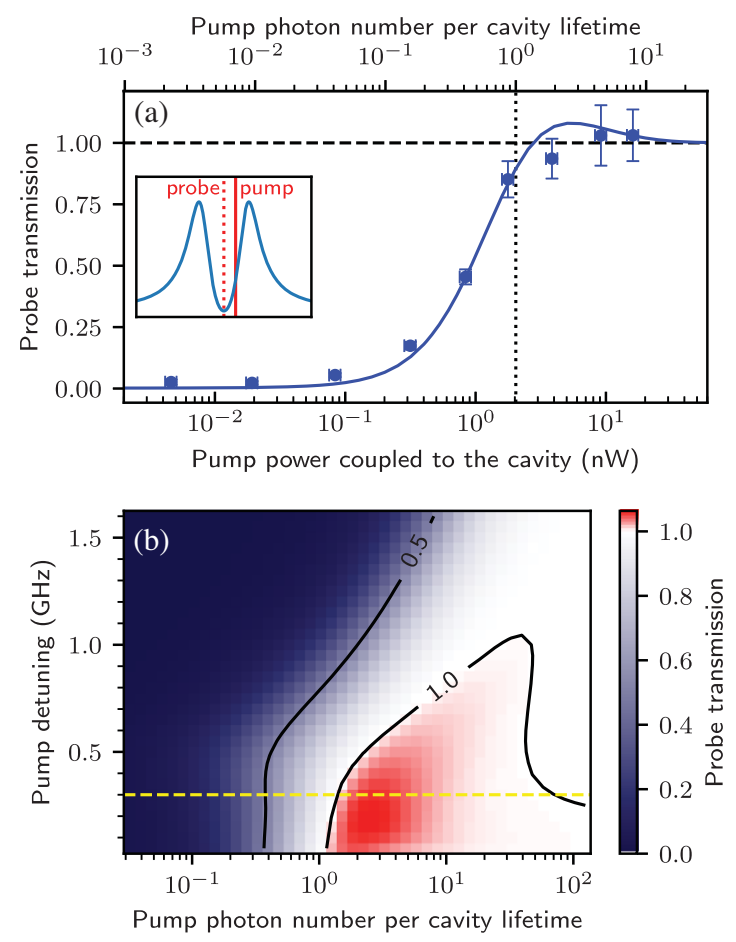

FIG. 4. (a) Transmission of the probe beam as a pump beam excites the molecule at a detuning of $300 \mathrm{MHz}$. The symbols and the solid curve present the experimental and theoretical data, respectively. Measurements close to full transmission involve smaller spectral features and thus lead to larger error bars. (b) Calculated transmission of a probe beam as a function of the pump frequency detuning from the cavity resonance and the pump power. The horizontal dashed line indicates the conditions for measurements presented in (a).

of pump and probe parameters, in Fig. 4(b) we present the calculated value of the probe transmission that can be achieved using our system parameters at different frequency detunings and pump powers. We find that it is advantageous to choose smaller frequency detunings for achieving switching at lower power, while keeping the frequency difference large enough to be able to separate them in the detection path with high fidelity.

Single-photon nonlinearities of quantum emitters have been considered for switching and quantum information processing [36]. Previous works have stated that perfect switching by single photons is not possible in two-level atoms due to a time-bandwidth issue and distortions of the photon wave packet $[37,38]$, although alternative arguments have also been put forth [39]. Our work inspires another intriguing approach, where single quantum emitters would be used as nano-optical logic elements for optical signal processing $[40,41]$ with very weak light fields of average photon number $\bar{N} \sim 1$. The organic solidstate platform presented in this Letter extends the pallet of material systems that have been used in quantum optics and offers significant advantages due to the ease of fabrication, availability in a wide range of wavelengths, brightness, and 
Fourier-limited emission [42]. The capacity of the organic matrices to host a very large density of dye molecules in the order of $10^{4}$ per $\mu \mathrm{m}^{3}$ makes molecular CQED easily extendable to a regime, where many emitters are strongly coupled to the same optical mode [43-45]. Such an arrangement could mediate nonlinear interactions [46] or generate $\mathrm{N}$-photon bundles [47] in integrated photonics circuits [48-50]. Single-molecule platforms can also be used for exploring a number of fundamental phenomena that have been predicted in the strong coupling regime of a two-level atom such as bistability [51,52], steadystate population inversion [53], and single-emitter lasing [54-57]. Furthermore, Fourier-limited coherence in organic molecules can be combined with plasmonic nanostructures [58] and hybrid architectures [59] to realize a nanoscopic realm of single-molecule strong coupling.

We thank Claudiu Genes and Dirk Englund for discussions, Jahangir Nobakht and Jan Renger for experimental support, and the Max Planck Society and the German Ministry of Research and Education (Grant No. 13N14839 within the research program "Photonik Forschung Deutschland") for financial support.

*Present address: Department of Physics, University of Regensburg, Universitätsstraße 31, D-93040 Regensburg, Germany.

${ }^{\dagger}$ Present address: Experimentalphysik I, Universität Kassel, Heinrich-Plett-Straße 40, D-34132 Kassel, Germany.

"Present address: LUMICKS B. V., Pilotenstraat 41, 1059CH Amsterdam, The Netherlands.

${ }^{\S}$ Present address: Departamento de Física Teórica de la Materia Condensada and Condensed Matter Physics Center (IFIMAC), Universidad Autónoma de Madrid, E28049 Madrid, Spain.

[1] S. Mukamel, Principles of Nonlinear Optical Spectroscopy, Oxford Series in Optical and Imaging Sciences (Oxford University Press, New York, 1999).

[2] R. W. Boyd, Nonlinear Optics (Elsevier, New York, 2003).

[3] G. Zumofen, N. M. Mojarad, V. Sandoghdar, and M. Agio, Perfect Reflection of Light by an Oscillating Dipole, Phys. Rev. Lett. 101, 180404 (2008).

[4] A. Maser, B. Gmeiner, T. Utikal, S. Götzinger, and V. Sandoghdar, Few-photon coherent nonlinear optics with a single molecule, Nat. Photonics 10, 450 (2016).

[5] R. Loudon, The Quantum Theory of Light, 3rd ed. (Oxford University Press, New York, 2000).

[6] R. J. Thompson, G. Rempe, and H. J. Kimble, Observation of Normal-Mode Splitting for an Atom in an Optical Cavity, Phys. Rev. Lett. 68, 1132 (1992).

[7] T. Yoshie, A. Scherer, J. Hendrickson, G. Khitrova, H. M. Gibbs, G. Rupper, C. Ell, O. B. Shchekin, and D. G. Deppe, Vacuum Rabi splitting with a single quantum dot in a photonic crystal nanocavity, Nature (London) 432, 200 (2004).
[8] D. Najer, I. Söllner, P. Sekatski, V. Dolique, M. C. Löbl, D. Riedel, R. Schott, S. Starosielec, S. R. Valentin, A. D. Wieck, N. Sangouard, A. Ludwig, and R. J. Warburton, A gated quantum dot strongly coupled to an optical microcavity, Nature (London) 575, 622 (2019).

[9] J. Kasprzak, S. Reitzenstein, E. A. Muljarov, C. Kistner, C. Schneider, M. Strauss, S. Höfling, A. Forchel, and W. Langbein, Up on the Jaynes-Cummings ladder of a quantum-dot/microcavity system, Nat. Mater. 9, 304 (2010).

[10] Y.-S. Park, A. K. Cook, and H. Wang, Cavity QED with diamond nanocrystals and silica microspheres, Nano Lett. 6, 2075 (2006).

[11] E. Janitz, M. K. Bhaskar, and L. Childress, Cavity quantum electrodynamics with color centers in diamond, Optica 7, 1232 (2020).

[12] P. D. Maker, R. W. Terhune, and C. M. Savage, IntensityDependent Changes in the Refractive Index of Liquids, Phys. Rev. Lett. 12, 507 (1964).

[13] F. P. Schäfer, W. Schmidt, and J. Volze, Organic dye solution laser, Appl. Phys. Lett. 9, 306 (1966).

[14] D. G. Lidzey, D. D. C. Bradley, M. S. Skolnick, T. Virgili, S. Walker, and D. M. Whittaker, Strong exciton-photon coupling in an organic semiconductor microcavity, Nature (London) 395, 53 (1998).

[15] P. Törmä and W.L. Barnes, Strong coupling between surface plasmon polaritons and emitters: A review, Rep. Prog. Phys. 78, 013901 (2015).

[16] R. Chikkaraddy, B. de Nijs, F. Benz, S. J. Barrow, O. A. Scherman, E. Rosta, A. Demetriadou, P. Fox, O. Hess, and J. J. Baumberg, Single-molecule strong coupling at room temperature in plasmonic nanocavities, Nature (London) 535, 127 (2016).

[17] D. Wang, H. Kelkar, D. Martín-Cano, D. Rattenbacher, A. Shkarin, T. Utikal, S. Götzinger, and V. Sandoghdar, Turning a molecule into a coherent two-level quantum system, Nat. Phys. 15, 483 (2019).

[18] A. A. L. Nicolet, C. Hofmann, M. A. Kol'chenko, B. Kozankiewicz, and M. Orrit, Single dibenzoterrylene molecules in an anthracene crystal: Spectroscopy and photophysics, Chem. Phys. Chem. 8, 1215 (2007).

[19] Z.S. Sadeq, R. A. Muniz, and J. E. Sipe, One- and twophoton absorption spectra of dibenzoterrylene, Phys. Rev. Mater. 2, 075202 (2018).

[20] D. Wang, H. Kelkar, D. Martín-Cano, T. Utikal, S. Götzinger, and V. Sandoghdar, Coherent Coupling of a Single Molecule to a Scanning Fabry-Perot Microcavity, Phys. Rev. X 7, 021014 (2017).

[21] See Supplemental Material at http://link.aps.org/ supplemental/10.1103/PhysRevLett.127.133603 for more details on the experimental procedure, the data analysis and the used theory model.

[22] S. Haroche and J.-M. Raimond, Exploring the Quantum: Atoms, Cavities, and Photons (Oxford University Press, New York, 2006).

[23] J. M. Fink, M. Göppl, M. Baur, R. Bianchetti, P. J. Leek, A. Blais, and A. Wallraff, Climbing the Jaynes-Cummings ladder and observing its nonlinearity in a cavity QED system, Nature (London) 454, 315 (2008). 
[24] L. Fleury, J.-M. Segura, G. Zumofen, B. Hecht, and U. P. Wild, Nonclassical Photon Statistics in Single-Molecule Fluorescence at Room Temperature, Phys. Rev. Lett. 84, 1148 (2000).

[25] G. Wrigge, I. Gerhardt, J. Hwang, G. Zumofen, and V. Sandoghdar, Efficient coupling of photons to a single molecule and the observation of its resonance fluorescence, Nat. Phys. 4, 60 (2008).

[26] H. J. Carmichael, R. J. Brecha, and P. R. Rice, Quantum interference and collapse of the wavefunction in cavity QED, Opt. Commun. 82, 73 (1991).

[27] A. González-Tudela, E. del Valle, and F. P. Laussy, Optimization of photon correlations by frequency filtering, Phys. Rev. A 91, 043807 (2015).

[28] D. E. Chang, A. S. Sørensen, E. A. Demler, and M. D. Lukin, A single-photon transistor using nanoscale surface plasmons, Nat. Phys. 3, 807 (2007).

[29] D. Witthaut, M. D. Lukin, and A. S. Sørensen, Photon sorters and QND detectors using single photon emitters, Europhys. Lett. 97, 50007 (2012).

[30] C. Cohen-Tannoudji, J. Dupont-Roc, and G. Grynberg, Atom-Photon Interactions (Wiley-VCH Verlag $\mathrm{GmbH}$, Weinheim, Germany, 1998).

[31] H. F. Hofmann and S. Takeuchi, Violation of local uncertainty relations as a signature of entanglement, Phys. Rev. A 68, 032103 (2003).

[32] K. E. Dorfman, F. Schlawin, and S. Mukamel, Nonlinear optical signals and spectroscopy with quantum light, Rev. Mod. Phys. 88, 045008 (2016).

[33] X.-L. Chu, S. Götzinger, and V. Sandoghdar, A single molecule as a high-fidelity photon gun for producing intensity-squeezed light, Nat. Photonics 11, 58 (2017).

[34] H. M. Gibbs, S. L. McCall, and T. N. C. Venkatesan, Optical bistability, Opt. News 5, 6 (1979).

[35] J. Hwang, M. Pototschnig, R. Lettow, G. Zumofen, A. Renn, S. Götzinger, and V. Sandoghdar, A single-molecule optical transistor, Nature (London) 460, 76 (2009).

[36] I. L. Chuang and Y. Yamamoto, Simple quantum computer, Phys. Rev. A 52, 3489 (1995).

[37] J. H. Shapiro, Single-photon Kerr nonlinearities do not help quantum computation, Phys. Rev. A 73, 062305 (2006).

[38] J. Gea-Banacloche, Impossibility of large phase shifts via the giant Kerr effect with single-photon wave packets, Phys. Rev. A 81, 043823 (2010).

[39] D. J. Brod and J. Combes, Passive CPHASE Gate via CrossKerr Nonlinearities, Phys. Rev. Lett. 117, 080502 (2016).

[40] J. Hardy and J. Shamir, Optics inspired logic architecture, Opt. Express 15, 150 (2007).

[41] J. Touch, Y. Cao, M. Ziyadi, A. Almaiman, A. MohajerinAriaei, and A.E. Willner, Digital optical processing of optical communications: Towards an optical turing machine, Nanophotonics 6, 507 (2017).

[42] C. Toninelli, I. Gerhardt, A. S. Clark, A. Reserbat-Plantey, S. Götzinger, Z. Ristanovic, M. Colautti, P. Lombardi, K. D. Major, I. Deperasińska, W. H. Pernice, F. H. L. Koppens, B. Kozankiewicz, A. Gourdon, V. Sandoghdar, and M. Orrit, Single organic molecules for photonic quantum technologies, Nat. Mater. (2021), https://doi.org/10.1038/s41563021-00987-4.
[43] H. R. Haakh, S. Faez, and V. Sandoghdar, Polaritonic normal-mode splitting and light localization in a onedimensional nanoguide, Phys. Rev. A 94, 053840 (2016).

[44] R. E. Evans, M. K. Bhaskar, D. D. Sukachev, C. T. Nguyen, A. Sipahigil, M. J. Burek, B. Machielse, G. H. Zhang, A. S. Zibrov, E. Bielejec, H. Park, M. Lončar, and M. D. Lukin, Photon-mediated interactions between quantum emitters in a diamond nanocavity, Science 362, 662 (2018).

[45] P. Samutpraphoot and T.Đorđević, P. L. Ocola, H. Bernien, C. Senko, V. Vuletić, and M. D. Lukin, Strong Coupling of Two Individually Controlled Atoms Via a Nanophotonic Cavity, Phys. Rev. Lett. 124, 063602 (2020).

[46] I. Carusotto and C. Ciuti, Quantum fluids of light, Rev. Mod. Phys. 85, 299 (2013).

[47] C. S. Muñoz, E. del Valle, A. G. Tudela, K. Müller, S. Lichtmannecker, M. Kaniber, C. Tejedor, J. J. Finley, and F. P. Laussy, Emitters of N-photon bundles, Nat. Photonics 8, 550 (2014).

[48] D. Rattenbacher, A. Shkarin, J. Renger, T. Utikal, S. Götzinger, and V. Sandoghdar, Coherent coupling of single molecules to on-chip ring resonators, New J. Phys. 21, 062002 (2019).

[49] A. Shkarin, D. Rattenbacher, J. Renger, S. Hönl, T. Utikal, P. Seidler, S. Götzinger, and V. Sandoghdar, Nanoscopic Charge Fluctuations in a Gallium Phosphide Waveguide Measured by Single Molecules, Phys. Rev. Lett. 126, 133602 (2021).

[50] P. Minzioni, C. Lacava, T. Tanabe, J. Dong, X. Hu, G. Csaba, W. Porod, G. Singh, A. E. Willner, A. Almaiman et al., Roadmap on all-optical processing, J. Opt. 21, 063001 (2019).

[51] C. M. Savage and H. J. Carmichael, Single atom optical bistability, IEEE J. Quantum Electron. 24, 1495 (1988).

[52] P. R. Rice and H. J. Carmichael, Single-atom cavity-enhanced absorption. I. Photon statistics in the bad-cavity limit, IEEE J. Quantum Electron. 24, 1351 (1988).

[53] M. Lindberg and C. M. Savage, Steady-state two-level atomic population inversion via a quantized cavity field, Phys. Rev. A 38, 5182 (1988).

[54] Y. Mu and C. M. Savage, One-atom lasers, Phys. Rev. A 46, 5944 (1992).

[55] K. An, J. J. Childs, R. R. Dasari, and M. S. Feld, Microlaser: A Laser with One Atom in an Optical Resonator, Phys. Rev. Lett. 73, 3375 (1994).

[56] M. Nomura, N. Kumagai, S. Iwamoto, Y. Ota, and Y. Arakawa, Laser oscillation in a strongly coupled singlequantum-dot-nanocavity system, Nat. Phys. 6, 279 (2010).

[57] J. McKeever, A. Boca, A. D. Boozer, J. R. Buck, and H. J. Kimble, Experimental realization of a one-atom laser in the regime of strong coupling, Nature (London) 425, 268 (2003).

[58] J. Zirkelbach, B. Gmeiner, J. Renger, P. Türschmann, T. Utikal, S. Götzinger, and V. Sandoghdar, Partial Cloaking of a Gold Particle by a Single Molecule, Phys. Rev. Lett. 125, 103603 (2020).

[59] B. Gürlek, V. Sandoghdar, and D. Martín-Cano, Manipulation of quenching in nanoantenna-emitter systems enabled by external detuned cavities: A path to enhance strong-coupling, ACS Photonics 5, 456 (2018). 